\title{
Using Online Presence to Improve Online Collaborative Learning
}

\author{
http://dx.doi.org/10.3991/ijet.v7iS1.1918 \\ Zoran Jeremić ${ }^{1}$, Nikola Milikić1, Jelena Jovanović ${ }^{\text {, Mirjana Brković }}{ }^{2}$ and Filip Radulović ${ }^{3}$ \\ ${ }^{1}$ University of Belgrade, Belgrade, Serbia \\ ${ }^{2}$ University of Kragujevac, Čačak, Serbia \\ ${ }^{3}$ Universidad Politécnica de Madrid, Madrid, Spain
}

\begin{abstract}
Social software tools have become an integral part of students' personal lives and their primary communication medium. Likewise, these tools are increasingly entering the enterprise world (within the recent trend known as Enterprise 2.0) and becoming a part of everyday work routines. Aiming to keep the pace with the job requirements and also to position learning as an integral part of students' life, the field of education is challenged to embrace social software. Personal Learning Environments (PLEs) emerged as a concept that makes use of social software to facilitate collaboration, knowledge sharing, group formation around common interests, active participation and reflective thinking in online learning settings. Furthermore, social software allows for establishing and maintaining one's presence in the online world. By being aware of a student's online presence, a PLE is better able to personalize the learning settings, e.g., through recommendation of content to use or people to collaborate with. Aiming to explore the potentials of online presence for the provision of recommendations in PLEs, in the scope of the OP4L project, we have develop a software solution that is based on a synergy of Semantic Web technologies, online presence and socially-oriented learning theories. In this paper we present the current results of this research work.
\end{abstract}

Index Terms - Online Presence, Personal Learning Environments, Educational Services, Ontologies.

\section{INTRODUCTION}

The widespread adoption of social software tools and their ability to provide support for diverse kinds of learning-related activities (e.g., collaborative writing, annotation, and sharing of diverse kinds of digital resources), were the main drivers towards the realization of the concept of Personal Learning Environments (PLEs) [1]. The notion of PLE assumes personal selection and aggregation of different, often Web-based tools and services into a learning environment customized to the needs and preferences of an individual learner. In a PLE, learning activities are not confined within the "walls" of one system/tool, thus enabling learners to make use of a wide diversity of digital resources (content, tools, and services) available on the Web. In addition, social software tools, as the core element of a PLE, provide support for finding the most suitable human resources, i.e., experts in any given topic and/or peers to collaborate with and build open learning communities. Finally, the notion of PLE assumes that a learner is in control of his/her learning process and empowers him/her with the ability to select, use and mash-up the resources that are most appropriate for his/her learning needs and preferences.

An important feature of almost any social software tool is that it supports users in maintaining their online presence. The notion of online presence encompasses a person's overall presence in the online world and is comprised of the posted status messages, availability indicators, geo-location, avatars, etc [2]. For a large majority of people, the main rationale for maintaining their presence in the online world is to keep some form of passive interaction with their peers. In a PLE, the notion of global online presence, i.e., student's online presence expressed on different tools integrated into his/her PLE, could be especially important. By giving students insights into their classmates' activities, availability for chat, information about work overload, emotional state, likes and dislikes, and all of that regardless of the particular tool they are using in the given moment, students' global online presence can provide those missing nonverbal cues typical for face-to-face interaction. This further increases students' awareness of each other and positively affects their willingness to collaborate [3]. However, there is still no systematic solution to exchanging and integrating online presence data from different social software tools. This is due to the differences in the online presence data kept by different tools as well as different representations of semantically identical data [4].

One of the key technical challenges for the realization of the PLE concept is exactly the integration of different tools and services a PLE should comprise [5]. A recent trend that promises to offer a solution to this problem relies on the use of Semantic Web technologies. These technologies enable the integration of data and knowledge originating from disparate and often heterogeneous sources (tools/services) and as such could allow for combining and exchanging data among the tools and services integrated in a PLE [15]. In addition, they could be applied for modeling the semantics of one's presence in the online world, with the final aim of enabling interoperability among services that collect and use online presence data.

In the scope of the OP4L (Online Presence for Learning, http://op4l.fon.bg.ac.rs) project we make use of Semantic Web technologies to unambiguously represent, integrate and make use of data about students online presence, their mutual interactions, as well as their interactions with learning resources. By analyzing these integrated data, we were able to get relevant information about a student's overall learning context in any particular mo- 
ment during his/her learning process. This provided us with a strong foundation for the development of educational services for recommendation of relevant learning resources (both digital and human).

Being aware that the technology, no matter how advanced it is, could not bring the desired learning results on its own, we explored different pedagogical approaches that could complement our technical solution. Having explored different modern learning theories, for reasons explained later in the paper (see Section II), we decided to base our work on the theories of social constructivism and connectivism.

This paper presents the work we have done thus far, in the scope of the OP4L project, aiming to facilitate and foster learning by leveraging the synergy of Semantic Web technologies, online presence and socially-oriented learning theories. We start a paper with a brief outline of learning theories to support collaborative learning in PLE and online presence (Section II). Section III gives an example usage scenario, whereas in Section IV OP4L solution is explained in detail. A brief description of the implementation details is given in Section V. We give a comparison with some related work in Section VI. Finally, we conclude with an overview of the empirical evaluation of the OP4L solution which is currently in progress, and a discussion of the expected implications of this work for the next generation of PLEs.

\section{BACKGROUND}

In recent years we are witnessing a rising interest in and acceptance of Vygotsky's Social Development Theory [6], connectivism [7] and other modern pedagogical theories, which argue for learners' active involvement in the learning process and construction of knowledge through social interactions. Vygotsky's theory is one of the foundations of social constructivism. According to him, social interactions play a fundamental role in the process of cognitive development of a student. Students are constructing new ideas based on their prior knowledge as well as social interactions with More Knowledgeable Others (MKO). The MKO (teacher, expert, other peer) refers to anyone who has a better understanding or ability level, with respect to a particular task or concept. In his learning theory, called connectivism, Siemens [7] emphasized the effect technology has on learning. According to Siemens, knowledge is gained by connecting with other individuals in a network through the use of appropriate technology.

PLEs incorporate the strengths of both constructivism and connectivism learning theories. Among other things, applying these theories in the context of a PLE requires information about students' presence in the online world: who is currently present in the learning environment, who is available for communication, who is busy and unavailable for other activities, etc. By providing additional data about learners' present state, online presence allows for more subtle personalization and higher-quality recommendation (e.g., not recommending collaboration with a peer who is currently busy and does not want to be disturbed). Learners' online presence data might also reveal their current real world location, thus allowing the system to give recommendations regarding peers who are nearby and could be contacted for an ad-hoc F2F meeting.

There are several significant studies which have explored the notion of social presence in online education and its implications on the learning process. The studies have shown that social presence is one of the most significant factors in distance education [9] in terms of improving instructional effectiveness, initiating in-depth discussions, promoting collaborative learning, as well as building of a group's online sixth sense and thus improving the group's cohesion [3]. Furthermore, researchers have found a relationship between social presence and student satisfaction in learning environments [10], [11], [12]. Strong relationship between students' perception of interactions with other students and quality and quantity of learning has been reported by Picciano [13].

Even though today's students are very active users of Web-based social software tools where they eagerly maintain their online presence, to the best of our knowledge, previous research work did not focus on online presence as a mean for improving students' social presence in online learning environments. Instead, the researchers who explored this area considered primarily discussion forums and IM tools integrated within Learning Management Systems as the means for establishing social presence in e-learning environments. As Web-based social software tools are becoming a prevalent way of communication between students today, our research is focused on investigating how students' online presence established on these tools could be leveraged for facilitating and even motivating learning activities within PLEs.

\section{SCENARIO OF USE}

In order to illustrate how we apply the proposed technical and pedagogical solution, in this section we will present a scenario involving a student named Tom who is using PLE and works with his classmates on a software design problem related to the Composite software design pattern [14]. The given problem has to be solved in a workshop-like manner by performing several predefined tasks: it starts with a brainstorming session, after which students create software designs based on the previously proposed and discussed conceptual solutions; finally, they evaluate and comment each-others solutions. The idea behind these activities is to foster students' creative thinking and collaborative knowledge building processes.

During the brainstorming session, as his fellow students, Tom is also supposed to present how he would solve the given problem, as well as comment on and rate ideas given by other students. In order to perform this task, he searches online repositories for information about the Composite software design pattern. Afterward, Tom is using a UML modeling tool enabling him to draw a solution to the given problem in the form of UML diagrams, and upload it to the Learning Management System (LMS) used for the course. From within the LMS, teachers and other students are able to assess Tom's solution and give him the feedback. Unfortunately, Tom got stuck with his assignment and would like to ask his peers for help, but he is not sure who is the most competent to ask. He would highly appreciate a recommendation regarding whom to contact for assistance.

In the proposed solution, Tom would be recommended to contact colleagues who, based on their user profiles, have enough knowledge of the Composite design pattern or other similar software design problems and would be able to help. Tom could look up for them on the LMS's online chat tool, but they might not be online there. How- 
ever, most probably they would be online on their favorite social networks - socializing with their friends on Facebook, following news updates on Twitter, wandering around the town but updating their location via Foursquare, etc. In the proposed solution, Tom's LMS is able to present him with this information. It is able to pull the online presence data of his peers from the popular social networks and display their availability for chat or their recent activity. If based on a peer's online status (e.g., custom message stating 'work overload'), the system identifies that he/she is busy, it would not recommend the given peer as a person to ask for help. Similarly, if a peer's online status indicates that he/she is available only for friends (e.g., custom message 'here only for friends') and Tom is not one of them, that peer would also not be recommended. Let's suppose that the system does manage to find a peer who is currently available, e.g., on Facebook; it would recommend Tom to contact that peer by sending him/her a message to his/her Facebook message inbox. If the system had not have access to the Tom's peers' online presence data, it would have made incorrect recommendation by potentially suggesting Tom to contact any of the knowledgeable peers even though they would not be able to help him.

\section{OP4L SOLUTION}

In this section, we present the OP4L solution which is designed to support students while collaboratively learning online. It also aims at making use of the students' online presence on Web-based social software tools as a mean for building and maintaining social presence in PLEs. In addition, the online presence data is used for making better recommendation of peers to contact for help.

The core of the OP4L solution is DEPTHS (Design Patterns Teaching Help System), an online learning environment for collaborative project-based learning of software design patterns [15]. By using a common ontological foundation (described in the next section), it integrates several existing educational systems and tools, namely: an LMS, a Domain Modeling tool, Online Repositories of Learning Resources and a Feedback-generation tool. Accordingly, DEPTHS could be considered as a PLE. In addition, DEPTHS comprises several context-aware educational services aimed at enriching and fostering learning processes by recommending appropriate learning content (e.g., Web pages, lessons or discussion forum postings), as well as fostering collaborative learning activities by bringing together students who are dealing with the same software problem or have experience in solving similar problems.

DEPTHS and the entire OP4L solution are built on a set of interlinked ontologies that allow for the formal representation of the data about students, learning activities, learning content and the interactions among these three key elements of a learning process. These ontologies are briefly introduced in the following subsection.

\section{A. Ontological Foundation}

LOCO [16] is a generic framework for the formal representation of the notion of learning context. Learning context is here defined as a specific learning situation characterized by a learning activity, the learning content that was used or produced during that activity, the partici- pants (students and/or teachers) who took part in the learning activity, and the environment where the activity occurred. Accordingly, LOCO is composed of several mutually connected ontologies which allow for detailed modeling of each of the aforementioned key components of the learning context. Following the Linked Data best practices, LOCO establishes connections with well-known and widely used ontologies such as the Dublin Core vocabulary (http://dublincore.org/documents/dces/), FOAF (Friend-Of-A-Friend, http://xmlns.com/foaf/spec/), and SIOC (Semantically Interlinked Online Communities, http://rdfs.org/sioc/spec/).

DEPTHS is relying on the LOCO's Learning Context Ontology for semantic representation of student's overall interaction with the learning content and other participants in learning activities. This data is then used by DEPTHS for performing context-aware retrieval of resources on software design patterns from online repositories and its own repository of software artifacts (which primarily contains artifacts produced by students and shared by the community).

For describing software design patterns and other concepts from the software engineering domain, SKOS (Simple Knowledge Organization Scheme, http://www.w3.org/ $\mathrm{TR} /$ skos-reference/) is chosen as the W3C's official recommendation for formal modeling of controlled vocabularies. This allows for easy extending of the domain vocabulary, publishing and linking it with other data on the Web. DEPTHS uses these domain vocabularies to annotate semantically relevant online resources and extract metadata that is used for finding resources appropriate for a student's current learning context.

The aim of the Online Presence Ontology (OPO) is to formally represent all fragments of a user's Online Presence description dispersed on a wide variety of social software tools and networks, and enable integration and exchange of those data [4]. Online Presence can be seen as dynamic part of user profiles in online services, as opposed to static parts, already well supported by the FOAF ontology. OPO has a high goal of enabling interoperability between applications that publish online presence data. In the OP4L solution, OPO is used for representing and unifying students' online presence over different tools and services integrated into DEPTHS. In particular, this is achieved through the OPO Server, the system we describe in the following subsection.

\section{B. Online Presence Ontology Server - OPOS}

OPOS acts as a hub capable of pulling one's online presence data from various social services. It stores that data and makes them accessible to the applications the user has selected as trusted ones. In learning settings, OPOS is used for providing online presence data for the potential peer collaborators the system has suggested.

OPOS enables its users to register their accounts on different social tools and services and assign the privileges to access their online presence data from those accounts. Periodically, OPOS pulls the data from the user's accounts and stores them in the local data repository (Fig. 1, steps 1 and 2). Since the majority of social tools and services support only certain aspects of one's online presence and that data are presented in different formats, OPOS relies on the OPO ontology to represent and store the online presence data in a common, semantic-rich data representation for- 


\section{USING ONLINE PRESENCE TO IMPROVE ONLINE COLLABORATIVE LEARNING}

mat. This way, it constructs user's global online presence. OPOS is also able to synchronize one's online presence description over user's accounts on different services by updating them with the latest update of the user's online presence (Fig. 1, steps 1-3). 2):

The architecture of OPOS consists of several parts (Fig.

- Social services (Fig. 2.A). Online presence data are part of every user profile on any online social network and instant messaging service. Depending on the type of a social service, online presence data are pulled from the service using either a plug-in developed for that purpose, or an API provided by the given service.

- OPOS Core (Fig. 2.B). The key component of OPOS is OPOS Core, which is in charge of managing all online presence data coming in to OPOS from social services. It has an access to the users' preferences, and stores the online presence data upon their arrival at the system.

- OPOS mediators (Fig. 2.C). Since every social service is specific in terms of the kind of data it provides and the ways offered for accessing that data, everysocial service has a specific mediator which serves as a bridge between the OPOS Core component and the service itself. It knows how to handle all data and any requests between OPOS and a specific social service.

- OPO data repository (Fig. 2.D). All online presence data are gathered and stored in the data repository in the form of RDF triples. This enables integration of users' online presence data from various social services across the Web at one place. Furthermore, SPARQL endpoint and RESTful services are provided which give the possibility for external accessing and querying over the data repository.

- OPOS web application (Fig. 2.E). Users are able to access OPOS through a web application, which by having a simple and intuitive interface enables them to easily specify which social services they want to include in OPOS. For every service, it is possible to declare whether a user wants to receive his/her online presence data from and/or send data to that service. That data will later be used by the OPOS Core when managing users' data.

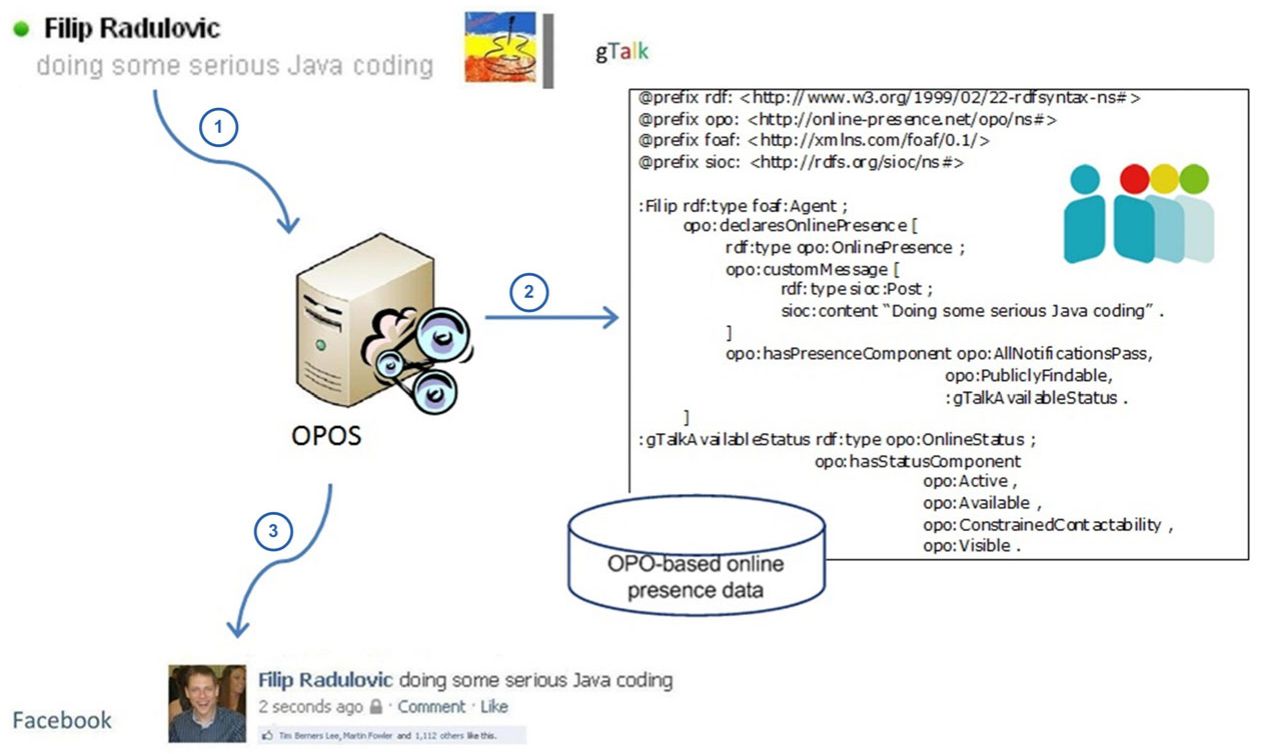

Figure 1. Data Flow in OPOS

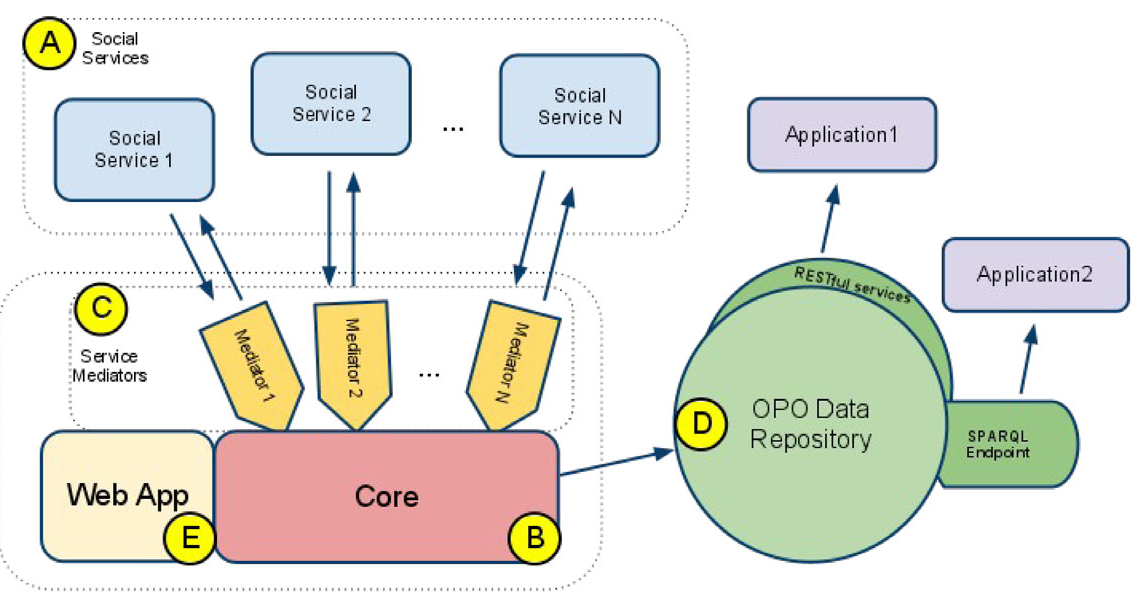

Figure 2. OPOS Architecture 


\section{SPECIAL FOCUS PAPER \\ USING ONLINE PRESENCE TO IMPROVE ONLINE COLLABORATIVE LEARNING}

The very fact that OPOS handles one's online presence data - considered by many as sensitive data - raises privacy concerns. This is something OPOS has yet to tackle as its primary focus is to materialize OPO's main idea of aggregating and exchanging online presence data coming from various services. A potential approach we are currently experimenting with is based on the oAuth protocol (http://oauth.net), a de facto standard authorization method with all popular social applications. This would provide users with the possibility to authorize other applications to access their data stored on the OPOS. The crucial challenge here will be to provide users with easily comprehensible and user-friendly interfaces that would allow them to set fine grained rules for data sharing and thus have full control over their personal data.

\section{Online Presence of Recommended Peers Module}

Online Presence of Recommended Peers is developed as a module of the LMS integrated into DEPTHS PLE. This module uses Peers Recommendation Service which is responsible for the creation of a list of users who are potentially relevant for the student's current learning context (the service is explained in the following section). For each peer selected as potentially relevant, a query is sent to the OPOS data repository to retrieve his/her latest online presence data. The retrieved data are used to provide visual indication (Fig. 3) of the peer's presence on different social tools and services integrated in the PLE.

When a student is in a need for contacting a colleague, e.g., to ask for help with the current assignment, he/she could take a look at the peers listed in the Online Presence of Recommended Peers Module and choose one of those whose online presence indicator suggests that he/she is free to be contacted. The chosen peer could be contacted directly within the social tool he/she is using in the given moment. A click on the online presence indicator initiates a message dialog for posting a request to the peer's social account (Fig. 4). To enable communication with a peer who is online on Facebook, a custom Facebook application called FB-OPOS has been developed.

The Online Presence of Recommended Peers module allows direct communication between LMS and Facebook provided that the following prerequisites are met:

- the given student has allowed Facebook application FB-OPOS to access his/her account;

- recommended peers are student's Facebook friends; if they are not friends, the system initiates Add friend request dialog;

- the student has not prohibited posting to its Wall in the Facebook privacy settings.

After receiving the notification message (in Facebook), a peer is able to respond by giving answers directly from his/her current context, i.e., he/she does not need to change the present learning/leisure context (Fig. 5).

In this case, the student who sent the initial request will be notified of the received response directly within the LMS (Fig. 6).

Besides being able to respond directly from Facebook, the peer is also given an option to visit the LMS and within it, the specific learning context from which the request has been sent.
Complete history of peers' communication (i.e., the exchanged messages) is kept and is available to the peers who participated in the communication on their request from Online Presence of Recommended Peers module. The history is organized by software design problems and LMS/social tool it was created in is indicated with a corresponding icon (Fig. 7).

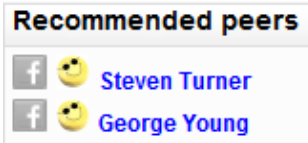

Figure 3. Online Presence of Recommended Peers Module

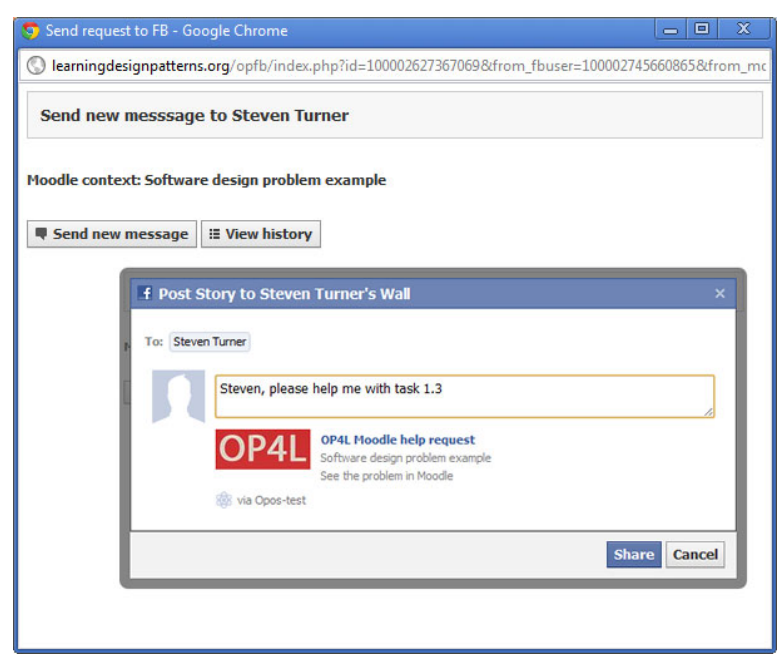

Figure 4. Sending message to peer's Facebook account

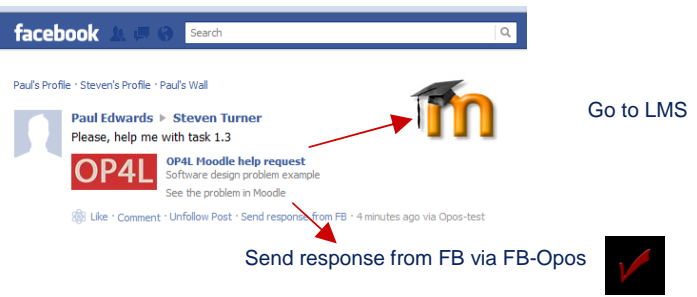

Figure 5. Answering the request directly in social tool

\section{DEPTHS demo course}

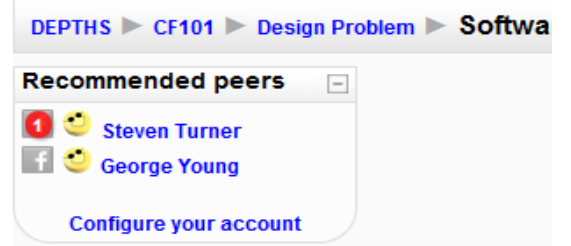

Figure 6. The notification about the received answer shown within the original learning context (i.e., LMS)

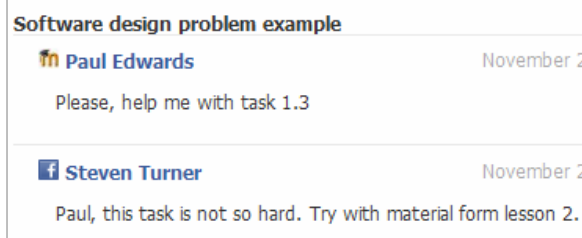

Figure 7. Peers' messages history example 
As it can happen that users are not that frequently using Facebook or not using it at all, we are currently working on an extension of the Online Presence of Recommended Peers module which will support communication with Twitter. From within the LMS integrated into the OP4L solution, students will be able to send direct messages on Twitter to their peers regarding their learning tasks. Likewise, they will be notified of responses to those messages, again within the LMS. Other services will be considered for making this kind of integration based on their popularity with students and ways of communication they offer (e.g., the next service we plan to integrate is Foursquare, https://foursquare.com/).

\section{Educational Services in $O P 4 L$}

Context-aware learning has gained an increasing attention in the e-learning research community recently. Despite different interpretations of the term 'context', researchers seems to agree that a learning context is about the environment, people, tools, resources and learning activities [16]. In this research, we slightly extend this notion of learning context to include also the users' online presence data.

In order to provide effective, context-aware learning DEPTHS offers the following educational services: Semantic Annotation and Indexing Service, Resource Recommendation Service and Peers Recommendation Service.

Semantic Annotation and Indexing Service is used for semantic annotation and indexing of online resources in publicly accessible repositories, as well as internally produced content (created by students). This module analyses text of each document, recognizes specific domain concepts defined in the domain ontology, finds what the document is about and how relevant it is for a specific domain concept. In order to find what the document is about, we used proven statistical measures, namely term frequency-inverse document frequency (TF-IDF) and cosine similarity [17]. TF-IDF is used to evaluate how important a word is to a document in a collection. This way, a collection of relevant documents is created for each domain topics. Afterwards, cosine similarity compares each document with an imaginary document (whose relevance is ideal), and sorts the documents in the collection based on their similarity, i.e. their relevance for a specific topic. Semantic annotation of the internally produced content is performed in a similar way.

Resource Recommendation Service employs student's current learning context, and generates a list of recommended Web resources or internally produced content. To do this, it computes the relevance for each resource for the student's current learning context and selects the most relevant Web resources or internal content (e.g., software projects) for the student. The computation of relevancy is based on the semantic metadata produced by Semantic Annotation and Indexing Service and formal representation of the student's current learning context represented in accordance with the LOCO's ontologies. The service also makes use of students' assessment of the resource's relevance for the current learning context (each time a student visits a suggested resource, he/she is asked to rate its relevance for the given context). Thus, students' ratings affect the resource's overall rating.
Peers Recommendation Service suggests other students, teachers or experts as possible collaborators. Collaborators are selected and sorted using an algorithm which estimates on three different levels their competence to provide help. The highest influence on the estimation of someone's competence to provide help has the estimated knowledge of the same topic, i.e. the ability to resolve the same software design problem the student needs help with. Smaller, but still high influence is given to someone's knowledge of similar or related topics. We believe that somebody is able to assist a student in need of help even if he/she hasn't solved the same problem, but has some experience with similar or related problems. The least influence is given to the knowledge of broader topics, e.g., ability to solve design problems that belong to the same course as the problem the student needs help with. On each of these levels, we are assessing three types of indicators:

1. the kind of learning activity one has participated in (e.g. brainstorming, submitting or assessing peers' work);

2. knowledge level as valued by the teacher and peers (including peers' evaluations of the submitted projects and ideas rating); and

3. social connections with the student asking for help the stronger social connection with a specific person, the higher is the estimation of the peer's competence to help student.

After the assessment of potential peers is performed, a list containing relevant peers sorted by their competences to help student in the current learning context is displayed (in the Online Presence of Recommended Peers module, Fig. 2).

Yet another feature provided for students in the OP4L environment is Social Streaming functionality, giving students a possibility to subscribe to a specific learning context, such as a software design problem, a brainstorming or a discussion thread. If a student decides to subscribe to a specific learning context, he/she will be notified about each social event happened in the selected learning context, e.g., a solution has been submitted for the problem being followed, a new post appeared in the discussion thread or a new idea was presented in the brainstorming thread of student's interest. The notification is in the form of a message sent automatically to the social tool he/she is using at the moment.

\section{OP4L IMPLEMENTATION}

To develop the OP4L solution, we used and further extended two frameworks developed in our previous research projects, namely DEPTHS [15] and OPOS (http://goodoldai.org/project opos). Within DEPTHS environment we have integrated the Moodle LMS and ArgoUML software modeling tool. Moreover, we use semantic annotation services of the KIM framework (http://www.ontotext.com/kim) to add semantic annotations (i.e., annotations with concepts from domain specific ontologies) to the online resources and internal content produced within the LMS. For the purposes of OP4L project we have extended DEPTHS with Facebook and Twitter integration components. As for OPOS, up to now, it supports the following social services: Facebook, Twitter, Foursqare and instant messaging client Spark. To communicate with these services, it uses their corresponding APIs. 


\section{SPECIAL FOCUS PAPER \\ USING ONLINE PRESENCE TO IMPROVE ONLINE COLLABORATIVE LEARNING}

Communication between all components within OP4L solution is enabled through the use of RESTful services implemented using the Jersey framework (http://jersey.java.net/). RDF repositories are implemented using Jena SDB (http://openjena.org/SDB/) which enables scalable storage and query of RDF data using relational databases. As our backend applications are based on the Java technology, we used Jenabean framework (http://code.google.com/p/jenabean/) for bidirectional mapping between RDF triples and Java OO model.

\section{RELATED WORK}

Having analyzed literature we found several research efforts related to the work described in this paper.

meNow Schema (http://crschmidt.net/semweb/menow) is a simple formal model allowing people to describe their current online status. However, this schema is relatively poor in capturing the semantics of status messages and states of availability.

In [18] a theoretical model for capturing the semantics of Presence in Social Networks has been suggested. Unlike other models, this model does not represent just the phenomenon of presence, but also emphasizes the presence data publishing and consumption aspects, as well as the purpose of the presence data. However, there is no information about the formal specification of this model.

An e-learning framework proposed in [19] supports recommendation of peers based on a student's context. The student's context is defined as a result of the interaction of three key elements: the knowledge potential, the social proximity and the technical access. Comparing to the OP4L's approach to recommendation of peers, the approach presented in [19] is advantageous as it considers technical context that includes factors such as technical media or time proximity. However, that approach does not consider the influence that a student's participation in the learning activities has on his competences to help other students, neither it considers students' online presence.

Graasp [20] is an online learning environment that allows users to aggregate data and content from different social media and social networking applications. It allows users to communicate and collaborate not only within the system boundaries, but also with peers who are using some external application for online communication or collaboration. However, it does not provide any kind of contextualization and recommendation of the peers, as it is the case in the OP4L solution.

\section{CONCLUSION}

In this paper, we have presented how students' online presence expressed on Web-based social software tools can be used within a PLE to support the establishment and maintenance of social connections among students as well as to enhance recommendation of peers who are not just eligible to help, but are also available in a particular moment. In order to achieve this, we made use of Semantic Web technologies to integrate and unify students' online presence data. These data are further fed into advanced educational services that leverage students' overall learning context to recommend them relevant learning resources (both digital and human). This work is a part of our broader research efforts, conducted within the scope of the OP4L project, to better support collaborative learning in personal learning environments.
An evaluation study of the developed software solution (i.e., the OP4L solution) is planned to be performed during the 2011/2012 academic year in high educational institutions of our four project partners. Overall, 240 students will take part in this study. The study is currently taking place in a college in Belgrade, Serbia, whereas the other three partners have scheduled the study for the spring semester 2012. The primary research questions to be investigated in this study are focused on the role that students' online presence plays in a PLE, namely, its influence on: 1) the perceived usefulness and usability of the peer recommendation service, and 2) students' overall learning experience. Moreover, we want to investigate if the students' motivation was affected by their perception of online presence, social tools and other educational services integrated in our PLE.

In order to involve students in the study, two software design tasks are defined as the exam prerequisites. Each task carries $25 \%$ of the final grade: $10 \%$ is given for the participation in each task and $15 \%$ for a completed task. In addition to these pre-exam tasks, students are asked to participate in four online surveys. In the first survey, we are using Motivated Strategies for Learning Questionnaire (MSLQ) in order to measure students' motivational orientations and learning strategies [21]. Two questionnaires, to be filled in by students after finishing each task, aim at investigating the students' perceptions of the learning activities, learning services and online presence provided within the DEPTHS PLE. The last survey, which should be performed after the competition of both tasks, aims to investigate the perceived usefulness and usability as well as the overall learning experience in the DEPTHS PLE. Students are given three weeks to perform each task, one week for each activity within a task (brainstorming, preparing and submitting proposal and evaluation of peers' proposals).

Given that the OP4L solution is in its first prototype version, our expectations are not so high. However, we are expecting to confirm our hypothesis that students perceive online presence as useful for facilitating collaboration and knowledge sharing, as well as stimulating active participation and building of group cohesion.

\section{REFERENCES}

[1] Attwell, G.: The Personal Learning Environments - the future of eLearning? eLearning Papers, 2(1). (2007).

[2] Stankovic, M., Jovanovic, J.: Online Presence in Social Networks. In: Proceedings of W3C Workshop on the Future of Social Networks, Barcelona (2009).

[3] Piezon, S., Donaldson, R.: Online Groups and Social Loafing: Understanding Student-Group Interactions, Online Journal of Distance Learning Administration, Volume VIII, Number IV, Winter (2005).

[4] Stankovic, M. Modeling Online Presence. In: Proceedings of the First Social Data on the Web Workshop, Karlsruhe, Germany, (2008).

[5] Jeremic, Z., Jovanovic, J., and Gasevic, D... Personal Learning Environments on Social Semantic Web. Submitted to Journal of Semantic Web. (2011).

[6] Wertsch, J., Sohmer, R.: Vygotsky on learning and development. Human Development. 38, 332-37.(1995). http://dx.doi.org/ $10.1159 / 000278339$

[7] Siemens, G.: Connectivism: A learning theory for the digital age. International Journal of Instructional Technology and Distance Learning 2(1). (2005). 


\section{SPECIAL FOCUS PAPER \\ USING ONLINE PRESENCE TO IMPROVE ONLINE COLLABORATIVE LEARNING}

[8] Zimmerman, B. J.: A Social Cognitive View of Self-Regulated Academic Learning. J. of Educational Psychology, Vol.81, No.3, (1989). http://dx.doi.org/10.1037/0022-0663.81.3.329

[9] Tu, C. H.: The Measurement of Social Presence in an Online Learning Environment, International Journal on E-Learning, vol.1, no.2, 34-45, (2002).

[10] Hostetter, C., \& Busch, M. Measuring up online: The relationship between social, presence and student learning satisfaction. Journal of Scholarship of Teaching and Learning, 6(2), 1-12. (2006).

[11] Russo, T., \& Benson, S.: Learning with invisible others: Perceptions of online presence and their relationship to cognitive and affective learning. Educational Technology \& Society, v8, no1, 5462, (2005).

[12] Swan, K., \& Shih, L. F.: On the nature and development of social presence in online course discussions. Journal of Asynchronous Learning Networks, 9(3), 115-136. (2005).

[13] Picciano, A.: Beyond student perceptions: issues of interaction, presence, and performance in an online course. Journal of Asynchronous Learning Networks, 6(1), 21- 40. (2002).

[14] Gamma, E., Helm, R., Johnson, R., Vlissides, J.: Design Patterns: Elements of Reusable Object-Oriented Software. Addison-Wesley, Reading, MA (1995)

[15] Jeremic, Z., Jovanovic, J., Gasevic, D.: An Environment for Project-based Collaborative Learning of Software Design Patterns. International Journal on Engineering Education, 27(1), 41-51 (2011).

[16] Jovanović, J., Gašević, D., Brooks, C., Devedžić, V., Hatala, M., Eap, T., Richards, G.: Using Semantic Web Technologies for the Analysis of Learning Content. IEEE Internet Computing, 11(5). (2007).

[17] Manning, C. D., Raghavan, P., Schütze, H.: Introduction to Information Retrieval. Cambridge University Press (2008).

[18] Wilson, S.: Presence in Social Networks. In: Hatzipanagos, S. \& Warburton, S. (Eds). Handbook of Research on Social Software and Developing Community Ontologies. IGI Global, Hershey PA. (2009). http://dx.doi.org/10.4018/978-1-60566-208-4.ch032

[19] Yanlin, Z and Yoneo, Y.: A Framework of Context Awareness support for peer recommendation in the e-learning context, British Journal of Educational Technology, 38(2), 2007, pp.197-210. (2007)

[20] Bogdanov, E., El Helou, S., Gillet, D., Salzmann, C. and Sire, S. (2010). Graaasp: a web 2.0 research platform for contextual recommendation with aggregated data.. Proceedings of the 28th International Conference on Human Factors in Computing Systems, CHI 2010, Extended Abstracts Volume, Atlanta, Georgia, USA, pp. 3523-3528.
[21] Pintrich, R. R., \& DeGroot, E. V. (1990). Motivational and selfregulated learning components of classroom academic performance, Journal of Educational Psychology, 82, 33-40. http://dx.doi.org/10.1037/0022-0663.82.1.33

\section{AUTHORS}

Zoran Jeremić is an Assistant Professor of Software Engineering in the Department of Simulations and Distance learning at the Military Academy in Belgrade, Serbia. His research interests are in the areas of semantic technologies, Web technologies, software engineering, technology-enhanced learning and personalized learning. He can be reached at http://zoranjeremic.org.

Nikola Milikić is a Teaching Assistant at FOS - School of Business Administration, University of Belgrade, Serbia. His research interests are within areas of Social Web, Semantic Web and TEL. He could be reached at: http://nikola.milikic.info/

Jelena Jovanović is an Assistant Professor with the Department of Software Engineering, FOS - School of Business Administration, University of Belgrade, Belgrade, Serbia. Her research interests are in the areas of semantic technologies, Web technologies, technologyenhanced learning, and knowledge management. She can be reached at http://jelenajovanovic.net.

Mirjana Brković is an Assistant at Information Technology Department, Technical Faculty Čačak, University of Kragujevac. Her specialties are e-learning, Web technologies, TEL, and personalized learning. She can be reached at http://www.tfc.kg.ac.rs/m brkovic.

Filip Radulović is a PhD student at the Computer Science Faculty, Technical University of Madrid (UPM). His research is mainly focused on Semantic Web and Semantic Technology evaluations. He can be reached at: http://filip.milstan.net/

This article is a modified version of a paper presented at the Second International Conference eLearning 2011, held in October 2011, at Metropolitan University in Belgrade, Serbia. Received 31 December 2011. Published as resubmitted by the authors 8 February 2012. The research presented in this paper is performed as a part of the OP4L project (project no. SEEERANETPLUS-115) supported by the SEE-ERA.NET PLUS Coordination and Support Action of the European Community. 